\title{
Features of network oscillations in data from single-channel neuronal recording
} Amir Assadi ${ }^{* 1}$, Arash Bahrami², Erwin Montgomery ${ }^{3}$ and Hamid Eghbalnia ${ }^{4}$

\author{
Address: ${ }^{1}$ Department of Mathematics, University of Wisconsin-Madison, Madison, WI 53706, USA, ${ }^{2}$ Graduate Program in Biophysics, University \\ of Wisconsin-Madison, Madison, WI 53706, USA, ${ }^{3}$ Department of Neurology, University of Wisconsin-Madison, Madison, WI 53706, USA and \\ ${ }^{4}$ Department of Biochemistry, University of Wisconsin-Madison, Madison, WI 53706, USA \\ Email: Amir Assadi* - ahassadi@facstaff.wisc.edu \\ * Corresponding author
}

from Sixteenth Annual Computational Neuroscience Meeting: CNS*2007

Toronto, Canada. 7-12 July 2007

Published: 6 July 2007

BMC Neuroscience 2007, 8(Suppl 2):PI3 I doi:I0.I |86/I47|-2202-8-S2-PI3 |

(c) 2007 Assadi et al; licensee BioMed Central Ltd.

We present a new algorithm for analysis of neuronal data from single-channel time-series. Using a novel view of stochastic geometry to obtain a realistic model of nonlinear oscillatory systems, we extract traces of multiple oscillations from single-electrode extracellular recordings obtained from a representative neuron. Empiric data from neuronal recording has supported the observation that oscillations are prevalent in dynamics of the brain [1]. The literature on mathematical and computational modeling of neuronal networks of coupled oscillators is rich and includes numerous examples of successful explanations of experimental data. A number of mathematical approaches to model oscillatory networks of neurons take a deterministic approach to modeling the dynamics of such sophisticated complex biological systems where noise is added, if at all, as a test of stability of the system and its behavior under small perturbations. There are possible situations where the dynamics of the system depends fundamentally on the actual non-stationary statistics and the transient nature of "noise" in the system. The most natural mathematical theories in this context involve nonlinear dynamics and probability theory, which has been the subject of research in describing networks as well as individual neurons. The key theory that enables us to achieve a mathematically rigorous synergy between an ideal geometric theory and the biological reality is a recent merging of stochastic analysis and ergodic theory, known as the theory of Random Dynamical Systems (RDS) whose systematic foundations are laid out in the seminal work of L. Arnold
[2]. The far-reaching ideas of RDS require a demanding technical mastery of stochastic analysis and ergodic. Our geometric and topological view will illustrate some of the remarkable theoretical and numerical achievements that RDS offers for biologically realistic modeling of nonlinear dynamics, while at the same time, it illuminates the ideas behind its algorithmic development.

\section{References}

I. Montgomery EB Jr: Dynamically coupled, high-frequency reentrant, non-linear oscillators embedded in scale-free basal ganglia-thalamic-cortical networks mediating function and deep brain stimulation effects. Nonlinear Studies 2004, I I:385-42I.

2. Arnold L: Random dynamical systems Springer-Verlag, Berlin; 1998. 